Case Report

\title{
A 64-Year-Old Woman with Chest Pain, Limb Weakness, and Endometrial Cancer
}

\author{
Simon Ponthus, ${ }^{1}$ Omar Kherad, ${ }^{1}$ Nicole Petriccioli, ${ }^{1}$ \\ Johannes Alexander Lobrinus, ${ }^{2}$ and Pierre-André A. Guerne ${ }^{3}$ \\ ${ }^{1}$ Internal Medicine Department, Hôpital de la Tour, Geneva, Switzerland \\ ${ }^{2}$ Department of Pathology, Geneva University Hospitals, Geneva, Switzerland \\ ${ }^{3}$ Department of Rheumatology, Geneva University Hospitals, Geneva, Switzerland \\ Correspondence should be addressed to Simon Ponthus; simon.ponthus@gmail.com
}

Received 3 February 2017; Accepted 22 February 2017; Published 8 March 2017

Academic Editor: Tamás Constantin

Copyright (C) 2017 Simon Ponthus et al. This is an open access article distributed under the Creative Commons Attribution License, which permits unrestricted use, distribution, and reproduction in any medium, provided the original work is properly cited.

Necrotizing autoimmune myopathy (NAM) is a rare subgroup of idiopathic inflammatory myopathies (IIM). This pathology usually affects proximal limb muscles and in some cases the myocardium. Patients usually display proximal limb weakness. Muscular biopsy is required to confirm the diagnosis. We report the case of a 64-year-old woman with an atypical first presentation of NAM, manifested by chest pain in the context of metastatic endometrial cancer. The diagnosis of NAM was however made when she returned a second time with proximal limb weakness. A treatment with prednisone was then initiated, to which rituximab was rapidly associated, beside a specific chemotherapy.

\section{Background}

NAM is part of the spectrum of the inflammatory myopathies [1]. It is characterized by muscle biopsy findings that include necrosis of muscle fibers with concomitant regeneration and low level inflammation, particularly mediated by macrophages [2]. At least 4 types of different etiologies of NAM are recognized: antisignal recognition particle (anti-SRP) antibody syndrome; a subgroup of statin-triggered autoimmune myositis, sometimes associated with anti-3-hydroxy3methylglutaryl-coenzyme A reductase antibodies (antiHMGCR); and paraneoplastic syndromes and viral infections, especially human immunodeficiency virus (HIV) [3]. Anti-SRP syndrome is a severe form of NAM characterized by a rapid progression and frequent myocardial involvement. Endometrial cancer related to NAM has not yet been described in the literature.

\section{Case Presentation}

A 64-year-old woman, known for a uterine clear-cell carcinoma (pTlaNOG3) and treated by hysterectomy two years ago, presented to the emergency room with chest pain. She had no cardiovascular risk factors except for her age. Troponins and creatine kinase (CK) levels returned elevated $(1383 \mathrm{ng} / \mathrm{L}$ and $8808 \mathrm{U} / \mathrm{L}$ resp.); the electrocardiogram was normal. Even though no significant coronary lesion was detected on the cardiac catheterization, a diagnosis of nonST elevation myocardial infarction (NSTEMI) was made. A treatment of atorvastatin $20 \mathrm{mg}$ and aspirin $100 \mathrm{mg}$ once a day was prescribed. During this hospital stay, a CT-scan revealed pulmonary and mediastinal masses. A biopsy confirmed metastases of endometrial origin and chemotherapy with Paclitaxel and Carboplatin was initiated. Two months later, her oncologist referred her for investigation of deterioration of health status. She complained about shoulder and thigh weakness over the previous few weeks, preventing her from climbing stairs and lifting objects. She also mentioned having difficulty swallowing (dysphagia). She did not describe paresthesia or any other neurological symptoms and no chest pain. Physical exam revealed global amyotrophy of limbs mostly proximal. Symmetrical severe paresis (M2/5) of all proximal muscles was observed, while strength of biceps and triceps was only slightly diminished (M4/M5) and conserved 
TABLE 1: Laboratory findings.

\begin{tabular}{lcc}
\hline Variable & Result & Reference range $^{*}$ \\
\hline Creatine kinase (U/L) & 10176 & $<170$ \\
Troponin T (ng/L) & 1198 & $<50$ \\
Creatine kinase-MB $(\mu \mathrm{g} / \mathrm{L})$ & 262.6 & $<5.0$ \\
Aldolase $(\mathrm{U} / \mathrm{L})$ & 67 & $1.20-8.80$ \\
Aspartate aminotransferase (U/L) & 438 & $10-35$ \\
Alanine aminotransferase (U/L) & 327 & $10-35$ \\
Lactate dehydrogenase (U/L) & 1187 & $<250$ \\
CRP $($ mg/L) & 5.0 & $<5.0$ \\
Creatinine $(\mu$ mol/L) & 33 & $44-80$ \\
Antisignal recognition particle (anti-SRP) & NA \\
Anti-HMG coA reductase (anti-HMGCR) & Positive \\
Antiacetylcholine receptor (anti-AChR) & Negative & NA \\
Antimuscle specific kinase (anti-MuSK) & Negative & NA \\
Antivoltage gate calcium channel (anti-VGCC) & Negative & NA \\
\hline
\end{tabular}

${ }^{*}$ Reference range for nonpregnant adults, with no medical conditions.

more distally (hands). No sensitive deficit was noticed; the rest of the neurological examination was normal. She had no noticeable cutaneous manifestation.

\section{Investigations}

Laboratory findings (Table 1) showed elevated CKs (10176 U/L), Aldolase (67 U/L), ASAT (438 U/L), ALAT (327 U/L), and LDH (1187 U/L) consistent with possible cellular muscular lysis. Troponins were also high (1198 ng/L). Antiacetylcholine receptor (anti-AChR) and muscle-specific kinase (anti-MuSK) antibodies for myasthenia gravis were negative. Lambert-Eaton myasthenic syndrome antibodies (voltage gate calcium channel (VGCC) antibodies) were also negative. Cerebral and spinal MRI did not show any metastasis or other lesion consistent with the symptoms and signs. The electromyoneurography exam of the deltoid was consistent with a myopathic tracing. A needle biopsy was performed in the right quadriceps femoris muscle. Scattered necrotic muscle fibers, myophagia, and regeneration were observed. Furthermore, the muscle fibers showed marked size variation with few internal nuclei. Fibrosis was minimal and no significant lymphocytic inflammatory infiltration was observed (Figure 1). All of this was consistent with the diagnosis of NAM, which was confirmed by the finding of positive antisignal recognition particle (anti-SRP) antibodies in the serum.

\section{Treatment}

Prednisone $1 \mathrm{mg} / \mathrm{kg}$ once a day was given during two month followed by a progressive reduction. Rituximab was rapidly introduced (500 $\mathrm{mg}$ once a week during 4 weeks) followed by methotrexate (15 mg once a week). She continued specific chemotherapy, as treatment of neoplasia was necessary in this possibly paraneoplastic symptomatology [4]. She also benefited from physiotherapy as exercise seems to be effective

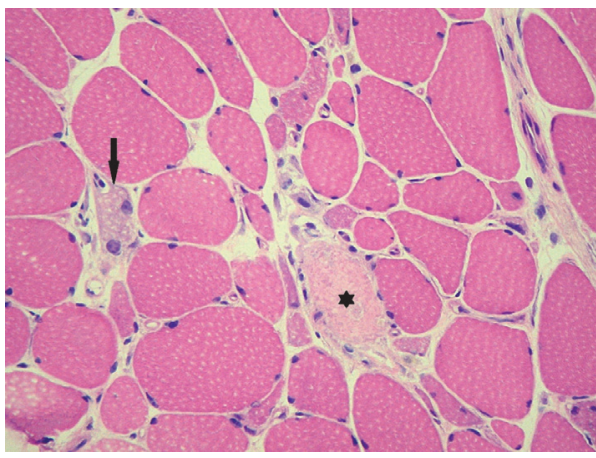

FIgUre 1: Muscle histology: frozen H\&E stained section, showing a necrotic muscle fiber (asterisk), with marked fiber size variation and regeneration (arrow), without lymphocytic infiltration (original magnification 200x).

in patients with IIM [5]. All treatments were well tolerated without major side effect.

\section{Outcome and Follow-Up}

Four months after the diagnosis and the beginning of treatments, we observed significant progress in her strength. She is now able to get up from her bed and walk without any help. Climbing stairs is still difficult but she also progressed in that point. Moving her shoulders and carrying objects is now easier. Rheumatologists of Geneva University Hospitals organized a follow-up.

\section{Discussion}

This case illustrates the complexity of NAM for which the etiological research can be challenging. This patient had endometrial cancer. Thus, the NAM could have been a paraneoplastic manifestation. Paraneoplastic NAM have been 
associated with gastrointestinal adenocarcinoma, small cell and non-small cell lung carcinoma, and breast and prostate cancers but, to the best of our knowledge, there is no case associated with endometrial cancer $[3,6]$. Moreover, serologic analyses revealed anti-SRP antibodies that are found in $16 \%$ of NAM [7]. These antibodies are more frequently found in women (sex ratio of 3.5) [8] and during fall season (possibly caused by a cross-reaction mechanism following a viral infection) [9]. We found one case reporting a patient with lung cancer and anti-SRP antibody [10] raising the question of a possible production of anti-SRP antibodies during neoplastic process.

Our patient initially came to the hospital with chest pain and elevated troponins and CK, but cardiac catheterization was normal. Retrospectively, this episode was most probably the first manifestation of her NAM. Indeed, cardiac involvement can be seen in NAM [1]; the exact incidence is unknown but the electrocardiogram might be abnormal in $50 \%$ of cases [8]. Exact consequences of cardiac involvement in NAM are unknown. After this cardiac episode, she received atorvastatin. This treatment could have enhanced the progression of the disease but does not seem to be responsible for it. In fact, the CK level was elevated before the introduction of statins. Furthermore, anti-HMGCR antibodies (a newly discovered autoantibody more frequently found in statin exposed patients) were not found in our patient [11]. Regardless of the etiology of NAM, prednisone is considered as the first-line therapy. Some patients, resistant to the initial therapy, could benefit from steroid-sparing agents such as methotrexate, azathioprine, intravenous immunoglobulin [9], or even rituximab [12]. Our patient accumulated poor prognosis factors [1] including associated malignancy, antiSRP antibodies, cardiac involvement, and dysphagia. We therefore introduced rapidly a relatively aggressive treatment, which proved, at least so far, well tolerated and associated with marked improvement and the possibility of decreasing considerably the potentially detrimental prednisone doses.

\section{Additional Points}

Learning Points. (i) NAM is a complex disease; several etiologies can coexist. (ii) Anti-SRP antibodies can also be found in paraneoplastic associated NAM. (iii) Chest pain with elevated troponins can be the first manifestation of NAM. (iv) Early recognition of poor prognosis factors could direct aggressive initial therapy.

\section{Conflicts of Interest}

All authors declare no conflicts of interest.

\section{References}

[1] I. N. Lazarou and P.-A. Guerne, "Classification, diagnosis, and management of idiopathic inflammatory myopathies," Journal of Rheumatology, vol. 40, no. 5, pp. 550-564, 2013.

[2] M. Sadeh and R. Dabby, "Steroid-responsive myopathy: immune-mediated necrotizing myopathy or polymyositis without inflammation?" Journal of Clinical Neuromuscular Disease, vol. 9, no. 3, pp. 341-344, 2008.
[3] C. Liang and M. Needham, "Necrotizing autoimmune myopathy," Current Opinion in Rheumatology, vol. 23, no. 6, pp. 612619, 2011.

[4] M. I. Levin, T. Mozaffar, M. T. Al-Lozi, and A. Pestronk, "Paraneoplastic necrotizing myopathy: clinical and pathologic features," Neurology, vol. 50, no. 3, pp. 764-767, 1998.

[5] G. E. A. Habers and T. Takken, "Safety and efficacy of exercise training in patients with an idiopathic inflammatory myopathy-a systematic review," Rheumatology, vol. 50, no. 11, pp. 2113-2124, 2011.

[6] B. Smith, "Skeletal muscle necrosis associated with carcinoma," The Journal of Pathology, vol. 97, no. 2, pp. 207-210, 1969.

[7] Y. Allenbach and O. Benveniste, "Acquired necrotizing myopathies," Current Opinion in Neurology, vol. 26, no. 5, pp. 554560, 2013.

[8] G. J. D. Hengstman, H. J. Ter Laak, W. T. M. Vree Egberts et al., "Anti-signal recognition particle autoantibodies: marker of a necrotising myopathy," Annals of the Rheumatic Diseases, vol. 65, no. 12, pp. 1635-1638, 2006.

[9] T. Miller, M. Al-Lozi, G. Lopate, and A. Pestronk, "Myopathy with antibodies to the signal recognition particle: clinical and pathological features," Journal of Neurology Neurosurgery and Psychiatry, vol. 73, no. 4, pp. 420-428, 2002.

[10] J. B. Sampson, S. M. Smith, A. G. Smith et al., "Paraneoplastic myopathy: response to intravenous immunoglobulin," Neuromuscular Disorders, vol. 17, no. 5, pp. 404-408, 2007.

[11] L. Christopher-Stine, L. A. Casciola-Rosen, G. Hong, T. Chung, A. M. Corse, and A. L. Mammen, "A novel autoantibody recognizing $200-\mathrm{kd}$ and $100-\mathrm{kd}$ proteins is associated with an immune-mediated necrotizing myopathy," Arthritis and Rheumatism, vol. 62, no. 9, pp. 2757-2766, 2010.

[12] R. Valiyil, L. Casciola-Rosen, G. Hong, A. Mammen, and L. Christopher-Stine, "Rituximab therapy for myopathy associated with anti-signal recognition particle antibodies: a case series," Arthritis Care \& Research, vol. 62, no. 9, pp. 1328-1334, 2010. 


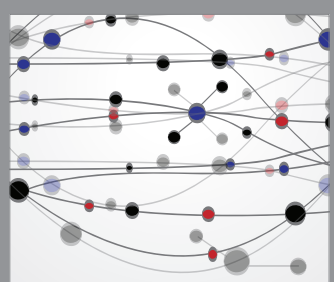

The Scientific World Journal
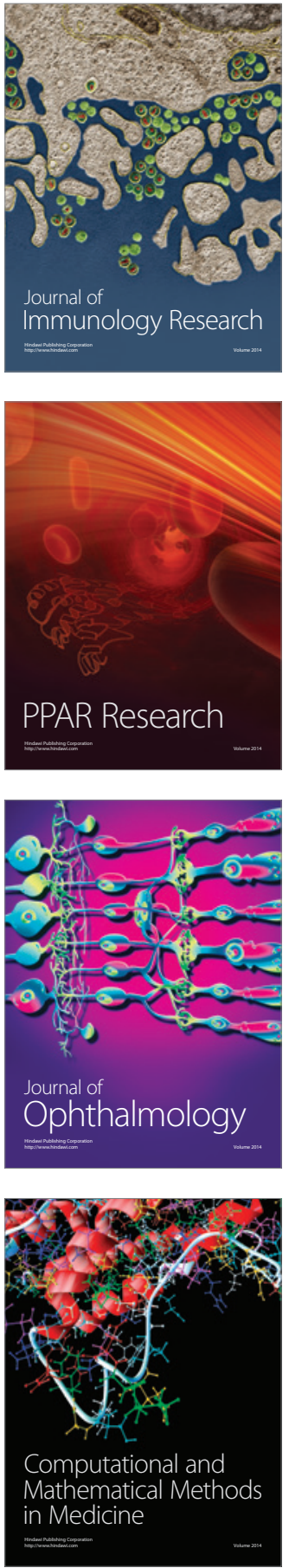

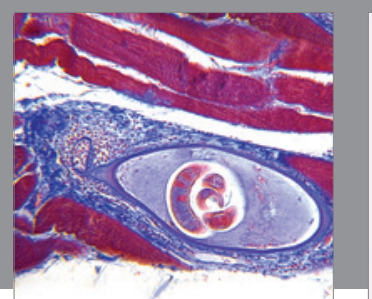

Gastroenterology Research and Practice
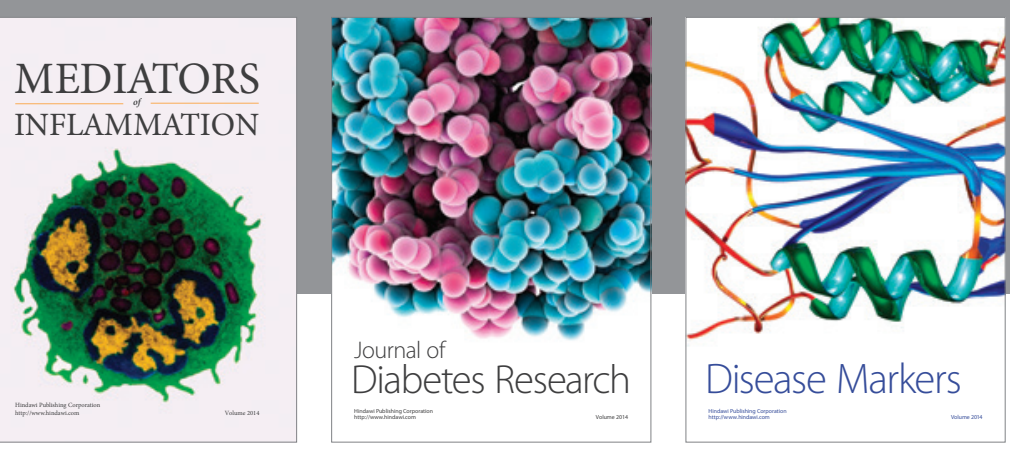

Disease Markers

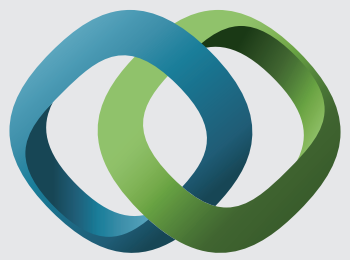

\section{Hindawi}

Submit your manuscripts at

https://www.hindawi.com
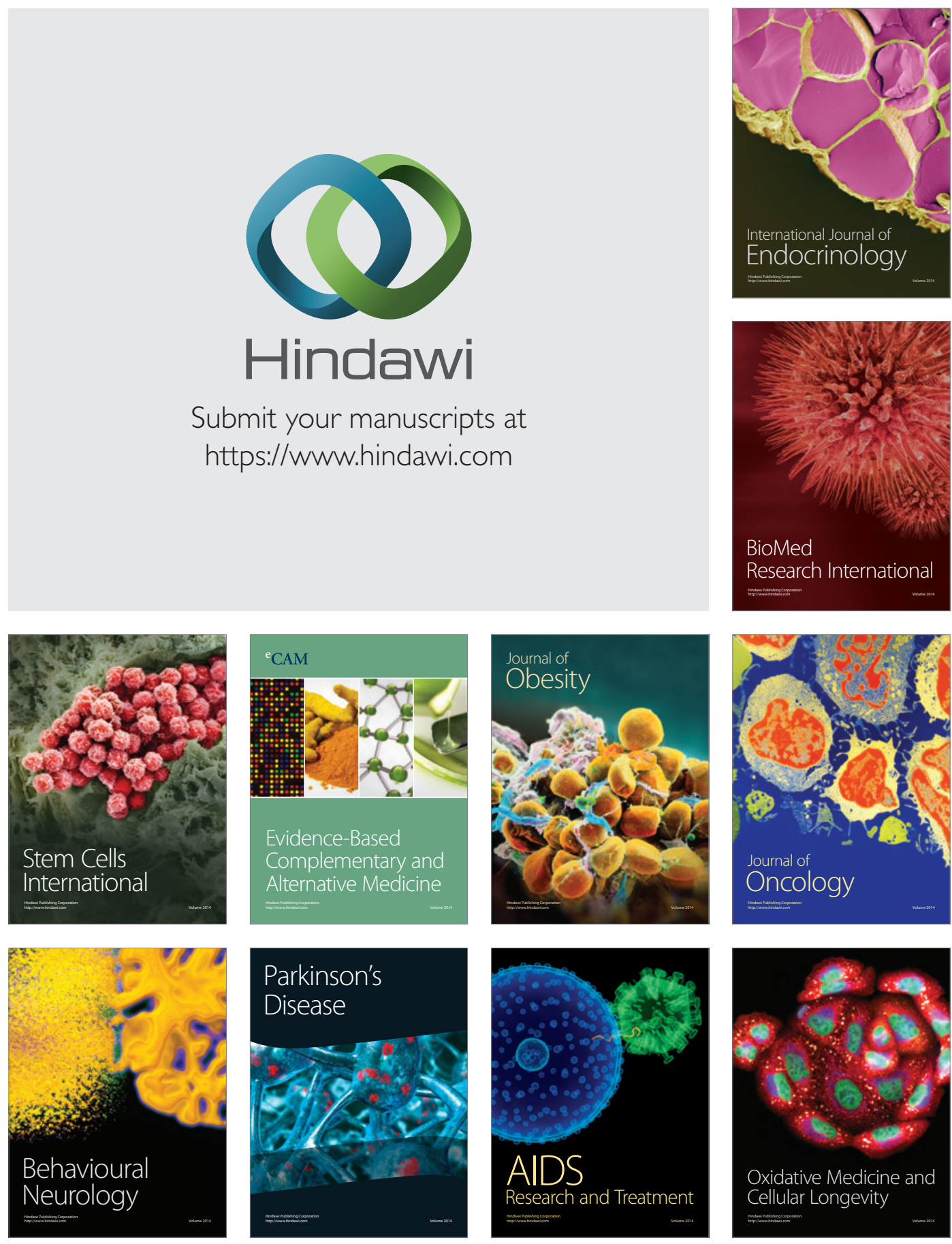